INPLASY

PROTOCOL

To cite: He et al. Risk factors for postanesthetic emergence delirium in adults: A systematic review and meta-analysis. Inplasy protocol 202210021. doi:

10.37766/inplasy2022.1.0021

Received: 05 January 2022

Published: 05 January 2022

Corresponding author:

Miao He

hemiao920@126.com

Author Affiliation:

Affiliated Hospital of Chengdu

University.

Support: Chengdu Health

Commission.

Review Stage at time of this submission: Preliminary searches.

Conflicts of interest:

None declared.

\section{Risk factors for postanesthetic emergence delirium in adults: $A$ systematic review and meta-analysis}

He, M1' Zhu, Z2; Jiang, M33; Liu, X4; Wu, R5; Zhou, J6.

Review question / Objective: Patientor population: patients with emergence delirium; Exposure: anaesthesia and surgery; Control: patients with no emergence delirium; Outcome: risk factors; Study design: meta-analysis.

Eligibility criteria: To ensure the quality of this meta-analysis, inclusion criteria was decided before we carried out the search. These criteria were: (a) Original researches that carried out in observational studies. (b)Adult patients who were extubated and recovered at PACU, operation room, or intensive care unit (ICU) after surgeries and anesthesia (including general and neuraxial anesthesia, peripheral nerve blocks and sedation). (c) Risk factors for delirium must be assessed with odds ratio (OR) with $95 \%$ confidence interval (CI). Researches must present the results of multivariate regression to be considered eligible for inclusion, since multivariate analysis results shall be used to identify variables eligible for meta-analysis. (d) Full-text available literatures.

INPLASY registration number: This protocol was registered with the International Platform of Registered Systematic Review and Meta-Analysis Protocols (INPLASY) on 05 January 2022 and was last updated on 05 January 2022 (registration number INPLASY202210021).

\section{INTRODUCTION}

Review question / Objective: Patientor population: patients with emergence delirium; Exposure: anaesthesia and surgery; Control: patients with no emergence delirium; Outcome: risk factors; Study design: meta-analysis.

Rationale: The risk factors and the mechanism of emergence delirium (ED) have not been elucidated. Clarifying the risk factors of ED is helpful to promote 
perioperative anesthesia management and improve surgical quality.

Condition being studied: Delirium is a common complication after surgery, which is characterized by acute and fluctuation alteration of mental state of reduced awareness and disturbance of attention. According to the onset time, delirium in the postoperative period can be classified as emergence delirium (ED) and postoperative delirium (POD). ED occurs during or immediately after emergence from general anesthesia or sedation; whereas POD is usually notable from postoperative day 1 and up to 1 week after surgery. ED, is considered to be the early stage of POD, can also present as hypoactive (decreased alertness, motor activity and anhedonia), as hyperactive (agitated and combative) or as mixed forms. However, there are no clear diagnostic criteria defining ED before nomenclature of perioperative neurocognitive disorders have been officially determined and released in 2018, which limiting its epidemiological researches. Initially, ED only refers to acute agitation, and now it is still often misused in the literature as a reference to "emergence agitation". Some scholars define it as "inadequate emergence", which is classified into two subtypes-emergence agitation and hypoactive emergence. The incidence of ED is dependent on multiple perioperative predisposing and precipitating factors, including patients' self-factors, anesthesia and surgery related factors. Strong evidence exists that patients with hyperactive form are at an increased risk of injury, hemorrhage, extended post-anesthesia care unit (PACU) stay, increased total hospital length of stay, morbidity, mortality, and resource utilization. However, hypoactive and mixed delirium has rarely been studied. The prognosis may be worse with hypoactive and mixed forms, possibly due to relative under-detection by staff and consequently delayed treatment. It would be ideal if we can early identify high-risk patients and give timely prevention and intervention accordingly. Unfortunately, the risk factors for adult ED have not been fully summarized. Though several observational studies assessed the risk factors for adult ED, single research or single-disease entity has no clinical guiding significance.

\section{METHODS}

Search strategy: Searches were conducted from onset of the databases until November 30, 2021 in electronic databases PubMed, Cochrane, and Embase (via OVID) with a search string comprising three concepts: "emergence delirium/acute delirium/emergence agitation/acute agitation/recovery room delirium" AND "surgery/surgical/operation/operative/ postoperative/postoperation" NOT "Pediatric/child/children". For every concept, relevant synonyms and subcategories were included in collaboration with an experienced biomedical librarian.

Participant or population: Patients with emergence delirium.

Intervention: Anaesthesia and surgery.

Comparator: Patients with no emergence delirium.

Study designs to be included: Cohort, cross-sectional and case-control studies.

Eligibility criteria: To ensure the quality of this meta-analysis, inclusion criteria was decided before we carried out the search. These criteria were: (a) Original researches that carried out in observational studies. (b)Adult patients who were extubated and recovered at PACU, operation room, or intensive care unit (ICU) after surgeries and anesthesia (including general and neuraxial anesthesia, peripheral nerve blocks and sedation). (c) Risk factors for delirium must be assessed with odds ratio (OR) with $95 \%$ confidence interval (Cl). Researches must present the results of multivariate regression to be considered eligible for inclusion, since multivariate analysis results shall be used to identify variables eligible for meta-analysis. (d) Full-text available literatures. 
Information sources: Information sources mainly come from PubMed, Cochrane, and Embase (via OVID). Any potentially relevant studies were manually searched based on the references of the identified trials and systematic reviews.

Main outcome(s): Independent risk factors of emergence delirium.

Quality assessment / Risk of bias analysis: New-castle Ottawa Quality Assessment Scale (NOS) for cohort and case-control studies, quality assessment for crosssectional study is recommended from Agency for Healthcare Research and Quality (AHRQ).

Strategy of data synthesis: Analyses were performed with Review Manager Software version 5.3. Meta-analysis was conducted when two or more studies reported similar independent risk factor. Forest plots were produced to visually assess the adjust ORs and corresponding $95 \%$ confidence intervals (Cls) for each risk factor, and chisquare tests were used for hypothesis testing ( $Z$ distribution, $P<0.05$, considered statistically significant). Heterogeneity among studies was measured with Cochran chi-square test and was considered present if $P<0.1$. Heterogeneity statistic 12 was used to indicate level of heterogeneity: we assign adjectives of low, moderate, and high to 12 values of $25 \%, 50 \%$, and 75 . The adjust ORs were first pooled using fixed-effect models. When $I 2>50 \%$ and $P<0.1$, indicating significant heterogeneity exists among included studies, random-effect model was incorporated.

Subgroup analysis: To explore the source of heterogeneity, subgroup analysis was conducted on the basis of existed features: studies with high quality and different screening scales for emergence delirium.

Sensitivity analysis: Sensitivity analysis was conducted by omitting one study in turn to examine the reliability and conclusiveness of the available evidence.
Keywords: emergence delirium, anaesthesia, surgery, adults, risk factors, meta-analysis.

Contributions of each author:

Author 1 - Miao He.

Email: hemiao920@126.com

Author 2 - Zhaoqiong Zhu.

Email: zhuzhaoqiong@zmu.edu.cn

Author 3 - Min Jiang.

Email: 176846022@qq.com

Author 4 - Xingxing Liu.

Email: 393948281@qq.com

Author 5 - Rui Wu.

Email: 172457746@qq.com

Author 6 - Junjie Zhou.

Email: 2763007929@qq.com

Country(ies) involved: China. 